\title{
Improvement of Tensile and Fatigue Properties of $\beta$-Titanium Alloy while Maintaining Low Young's Modulus through Grain Refinement and Oxygen Addition
}

\author{
Ken $\mathrm{Cho}^{1, *}$, Mitsuo Niinomi ${ }^{1}$, Masaaki Nakai ${ }^{1}$, Junko Hieda ${ }^{1}$ and Ryota Kanekiyo ${ }^{2}$ \\ ${ }^{1}$ Institute for Materials Research, Tohoku University, Sendai 980-8577, Japan \\ ${ }^{2}$ Department of Metallurgy, Materials Science, and Materials Processing, Tohoku University, Sendai 980-8579, Japan
}

To investigate methods of improving the tensile and fatigue properties of a solutionized Ti-29Nb-13Ta-4.6Zr (TNTZ) alloy without increasing its Young' modulus, two types of TNTZ alloys having oxygen contents of 0.06 and 0.14 mass\% (TNTZ-0.06O and TNTZ-0.14O), respectively, were subjected to cold swaging and a subsequent heat-treatment. The effects of the grain refinement caused by the cold swaging and the subsequent heat-treatment as well as those of oxygen addition on the microstructures, Young's moduli and tensile and fatigue properties of the two alloys were investigated.

The grain diameters of the TNTZ- $0.06 \mathrm{O}$ and TNTZ-0.140 decrease from $27 \mu \mathrm{m}$ (as-received) to $1.7 \mu \mathrm{m}$ and from $33 \mu \mathrm{m}$ (as-received) to $1.0 \mu \mathrm{m}$, respectively, after subjected to cold swaging and the subsequent heat-treatment. These results suggest that cold swaging, followed by heat treatment, is effective in refining the grains of TNTZ alloys. However, a $\beta$ (110) texture develops in the alloys as a result of the cold swaging.

Young's moduli of as-cold swaged and heat-treated TNTZ- $0.06 \mathrm{O}$ and TNTZ-0.14O are within the range of $61-68$ GPa and as low as those of solutionized TNTZ- $-0.06 \mathrm{O}$ and TNTZ-0.14O

The tensile strengths and elongations of the as-cold swaged, heat-treated and solutionized TNTZ- $0.14 \mathrm{O}$ are approximately $30 \%$ higher and $20 \%$ lower, respectively, than those of the corresponding TNTZ-0.06O. Moreover, the $0.2 \%$ proof stresses of the heat-treated TNTZ-0.14O are approximately $110 \%$ higher than that of the corresponding TNTZ-0.06O. On the other hand, the values of the Hall-Petch constant ( $k$ ) for the TNTZ-0.06O $\left(k_{\mathrm{TNTZ}-0.06 \mathrm{O}}=0.02\right)$ and TNTZ-0.14O $\left(k_{\mathrm{TNTZ}-0.14 \mathrm{O}}=0.005\right)$ are much smaller than those for pure Ti and another $\beta$-type Ti alloy (Ti-15.2Mo). These results indicate that the addition of oxygen can improve the tensile properties of TNTZ alloys. However, the grain refinement caused by cold swaging and a subsequent heat-treatment does not have a significant effect on the tensile properties of TNTZ.

The fatigue limit of the heat-treated TNTZ-0.14O (540 MPa) is much higher than those of the heat-treated and solutionized TNTZ-0.06O $(290$ and $230 \mathrm{MPa})$ and solutionized TNTZ-0.140 (330 MPa). These results indicated that it is possible to improve the fatigue properties of solutionized TNTZ using grain refinement, which can be induced by cold swaging and a subsequent heat-treatment and by the addition of oxygen. [doi:10.2320/matertrans.M2013151]

(Received April 18, 2013; Accepted July 4, 2013; Published August 23, 2013)

Keywords: metallic biomaterials, $\beta$-type titanium alloy, grain refinement, low Young's modulus, tensile and fatigue properties

\section{Introduction}

Young's modulus is one of the most important parameters for evaluating the suitability of metallic biomaterials for implant devices. This is because a large mismatch between Young's modulus of the implant material and that of cortical bone can cause stress shielding, resulting in inhomogeneous stress transfer between the bone and the implant device. ${ }^{1)}$ This can lead to bone atrophy, resulting in the loosening of the implant device and refracturing of the bone. A number of $\beta$-type titanium alloys, including the alloys $\mathrm{Ti}-29 \mathrm{Nb}-13 \mathrm{Ta}-$ 4.6Zr (mass \%, TNTZ) ${ }^{2)}$ and $\mathrm{Ti}-24 \mathrm{Nb}-4 \mathrm{Zr}-7.9 \mathrm{Sn}$ (mass\%), have been developed as low Young's modulus alloys in order to overcome these problems. Young's modulus of solutionized TNTZ is approximately $60 \mathrm{GPa},{ }^{4}$ ) which is much closer to that of cortical bone $\left.(10-30 \mathrm{GPa})^{5}\right)$ than are those of pure Ti (CP-Ti: $105 \mathrm{GPa})^{6)}$ and $(\alpha+\beta)$-type Ti alloys (Ti-6Al-4V ELI (Ti64 ELI): $110 \mathrm{GPa}){ }^{7)}$ Therefore, $\beta$-type Ti alloys are expected to be suitable materials for implant devices.

However, the tensile strength and fatigue limit of solutionized TNTZ are lower than those of Ti64 ELI, which is the most widely used alloy for biomedical applications, including for implant devices. The tensile strengths of solutionized TNTZ and aged Ti64 ELI are approximately $600 \mathrm{MPa}^{4)}$ and $1050 \mathrm{MPa},{ }^{7)}$ respectively. The fatigue limit of

*Corresponding author, E-mail: k_cho@imr.tohoku.ac.jp solutionized TNTZ is $320 \mathrm{MPa}^{4)}$ and that of aged Ti64 ELI ranges from $610 \mathrm{MPa}$ (in the case of the acicular $\alpha$ phase) to $800 \mathrm{MPa}$ (in the case of the equiaxed $\alpha$ phase). ${ }^{8)}$ Several studies have reported that the tensile strength of solutionized TNTZ can be increased by subjecting it to a cold rolling process. The reported tensile strength of cold rolled TNTZ is $800 \mathrm{MPa} .{ }^{9)}$ However, the fatigue properties of solutionized TNTZ are not improved by the cold rolling process. ${ }^{4)}$ On the other hand, aged TNTZ shows excellent tensile strength and fatigue properties because of precipitation strengthening by the $\omega$ and/or $\alpha$ precipitated phase. ${ }^{4,9}$ However, Young's modulus of aged TNTZ is higher than $85 \mathrm{GPa}$ because of the formation of the $\omega$ and/or $\alpha$ precipitated phase. ${ }^{4,9)}$ Therefore, new methods for improving the tensile and fatigue properties of solutionized TNTZ without increasing its Young' modulus are required.

Grain refinement strengthening is an effective method for improving the tensile properties of Ti alloys. ${ }^{10,11)}$ Moreover, by controlling the grain refinement processes, it is possible to keep the phase constitutions of the alloys unchanged. Therefore, one can assume that the grain refinement strengthening of solutionized TNTZ should have no effect its Young's modulus. In addition, it is known that the solid solution strengthening of $\mathrm{Ti}$ alloys with oxygen can improve their tensile strengths significantly. ${ }^{12,13)}$ Young's modulus of a metallic material is not only dependent on its phase constitution but also on the interatomic distance in the crystal 
Table 1 Chemical compositions of hot forged TNTZ-0.06O and TNTZ0.140 .

\begin{tabular}{cccccccc}
\hline Material & $\mathrm{Ti}$ & $\mathrm{Nb}$ & $\mathrm{Ta}$ & $\mathrm{Zr}$ & $\mathrm{C}$ & $\mathrm{N}$ & $\mathrm{O}$ \\
\hline TNTZ-0.06O & Bal. & 29.4 & 13.0 & 4.6 & 0.013 & 0.008 & 0.06 \\
TNTZ-0.14O & Bal. & 28.7 & 12.7 & 4.4 & 0.006 & 0.007 & 0.14 \\
\hline
\end{tabular}

lattice of the material. Therefore, the addition of oxygen may decrease the interatomic distance, resulting in an increase in its Young's modulus. However, Geng et al. have reported that if the oxygen content is less than 0.33 mass $\%$, the effect of oxygen addition on Young's modulus of TNTZ is insignificant. ${ }^{14)}$ Therefore, grain refinement and the addition of oxygen are expected to improve the tensile and fatigue properties of solutionized TNTZ without increasing its Young's modulus.

In this study, two types of TNTZ having different oxygen contents were subjected to a cold swaging process and a subsequent heat-treatment for grain refinement. The microstructures, Young's moduli and tensile and fatigue properties of these TNTZ were investigated.

\section{Experimental Procedures}

\subsection{Material preparation}

The chemical compositions of the two types of hot forged TNTZ (as received) having different oxygen contents used in this study are listed in Table 1 . The TNTZ containing 0.06 mass $\%$ oxygen is denoted as TNTZ $-0.06 \mathrm{O}$ and that containing 0.14 mass $\%$ oxygen as TNTZ- 0.140 . The diameters of the bars used of TNTZ- $0.06 \mathrm{O}$ and TNTZ-0.14O were 20 and $25 \mathrm{~mm}$, respectively. The oxide layer formed on the bars was removed mechanically before the bars were subjected to cold swaging, followed by heat-treatment.

\subsection{Cold swaging and heat-treatment processes}

Figure 1 shows a schematic drawing of the cold swaging and heat-treatment processes employed in this study. The diameters of the TNTZ- $0.06 \mathrm{O}$ and TNTZ- $0.14 \mathrm{O}$ bars were reduced from 20 to $5.5 \mathrm{~mm}$ and from 25 to $6.5 \mathrm{~mm}$, respectively, (with the reduction ratio being 92\%) through the cold swaging process, which was performed at room temperature in air. The cold swaged TNTZ-0.06O and TNTZ-0.14O bars were then subjected to heat-treatment at 923 or $973 \mathrm{~K}$ for 3.6 or $10.8 \mathrm{ks}$ in vacuum and were then quenched into water. Moreover, for comparison, cold swaged bars of TNTZ $-0.06 \mathrm{O}$ and TNTZ- $0.14 \mathrm{O}$ were subjected to a solution treatment at $1063 \mathrm{~K}$ for $3.6 \mathrm{ks}$ in vacuum, followed by quenching in water.

\subsection{Material characterization}

The microstructures of cross sections of the as-received, as-cold swaged, heat-treated and solutionized TNTZ-0.06O and TNTZ-0.14O bars were evaluated using an optical microscopy (OM). The specimens, which were cut from each bar into round slices, for the OM observations were wetpolished using waterproof emery papers of up to \#4000 grit and buff polished using a colloidal $\mathrm{SiO}_{2}$ suspension. They were then etched using a 5\% HF etching solution. The grain

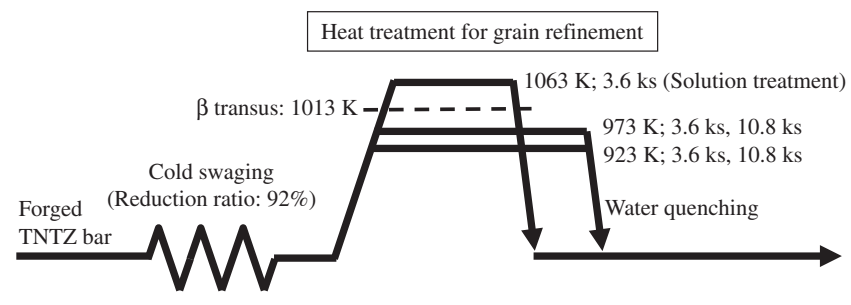

Fig. 1 Schematic drawing of cold swaging and heat-treatment processes employed in this study.

diameters of the as-received, heat-treated and solutionized TNTZ-0.06O and TNTZ-0.14O were quantified from the OM images using the average grain intercept (AGI) method.

The phase constitutions of cross sections of the asreceived, as-cold swaged, heat-treated and solutionized TNTZ $-0.06 \mathrm{O}$ and TNTZ- 0.140 bars were investigated by $\mathrm{X}$-ray diffraction (XRD) analyses. The specimens, which were cut from each bar into round slices, for the XRD analyses were wet-polished using waterproof emery papers of up to \#4000 grit. In addition, the phase constitutions of the fractured sections of the heat-treated and solutionized TNTZ- $0.06 \mathrm{O}$ and TNTZ-0.14O after subjected to a fatigue test were also investigated directly, i.e., without being polished, by XRD analyses. The XRD analyses were performed using a $\mathrm{Cu}$ target, with an operation voltage of $40 \mathrm{kV}$ and a tube current of $40 \mathrm{~mA}$.

The microstructures of the fractured sections of the heattreated and solutionized TNTZ-0.06O and TNTZ-0.14O after subjected to a tensile test were evaluated by transmission electron microscopy (TEM). The specimens used for the TEM observations were cut from the fractured sections of the samples subjected to the tensile test. These specimens were wet-polished using waterproof emery papers of up to \#4000 grit and then dimpled with a phosphor bronze ring to form dimples up to $30 \mu \mathrm{m}$ in size. Finally, these specimens were ion milled to form thin foils. The TEM observations were performed at an accelerating voltage of $200 \mathrm{keV}$.

\subsection{Mechanical properties}

Young's moduli of the as-cold swaged, heat-treated and solutionized TNTZ-0.06O and TNTZ-0.14O were measured using a free resonance method. Young's modulus measurements were made at room temperature in air. For the measurements, bars of TNTZ- $0.06 \mathrm{O}$ and TNTZ- $0.14 \mathrm{O}$ with a length of $50 \mathrm{~mm}$ and diameters of 5 and $6 \mathrm{~mm}$, respectively, were used. These specimens were wet-polished using waterproof emery papers of up to \#1500 grit.

The tensile properties of the as-cold swaged, heat-treated and solutionized TNTZ-0.06O and TNTZ-0.14O were evaluated at room temperature in air using an Instron-type testing machine that had a cross-head speed of $8.33 \times$ $10^{-6} \mathrm{~m} \cdot \mathrm{s}^{-1}$. For the tensile test, dog bone-shaped specimens with a gage length of $11 \mathrm{~mm}$ and a gage diameter of $3 \mathrm{~mm}$ were used. These specimens were wet-polished using waterproof emery papers of up to \#1500 grit.

The fatigue properties of the heat-treated and solutionized TNTZ- $0.06 \mathrm{O}$ and heat-treated TNTZ- 0.140 were evaluated using an electro-servo-hydraulic testing machine. The fatigue tests were performed at a frequency of $10 \mathrm{~Hz}$, with a stress 
ratio, $R$, of 0.1 , under the tension-tension mode at room temperature in air. The maximum cyclic stress, i.e., the stress at which the specimen being tested did not undergo failure after $1 \times 10^{7}$ cycles, was defined as the fatigue limit (run out). For the fatigue test, dog bone-shaped tensile specimens with a gage length of $11 \mathrm{~mm}$ and a gage diameter of $3 \mathrm{~mm}$ were used. These specimens were wet-polished using waterproof emery papers of up to \#4000 grit and buff polished using a colloidal $\mathrm{SiO}_{2}$ suspension.

\section{Results and Discussion}

\subsection{Microstructures and phase constitutions}

Figure 2 shows typical OM images of cross sections of the as-received, as-cold swaged, heat-treated and solutionized (a) TNTZ-0.06O and (b) TNTZ-0.14O bars. As can be seen from Fig. 2, it is difficult to observe the grain boundaries of the as-cold swaged TNTZ-0.06O and TNTZ-0.14O. This result suggests that plastic flow is caused in them by the cold swaging process. After the heat-treatment at $923 \mathrm{~K}$ for $3.6 \mathrm{ks}$, clear grain boundaries appear in the TNTZ-0.06O and TNTZ-0.14O specimens. The grain diameters of the asreceived, heat-treated and solutionized TNTZ-0.06O and TNTZ-0.14O quantified from the OM images, are shown in Fig. 3 as functions of the heat-treatment time. The grain diameters of the TNTZ- $0.06 \mathrm{O}$ and TNTZ- $0.14 \mathrm{O}$ heat-treated at $923 \mathrm{~K}$ for $3.6 \mathrm{ks}$ decrease from $27 \mu \mathrm{m}$ (as-received) to $1.7 \mu \mathrm{m}$ and from $33 \mu \mathrm{m}$ (as-received) to $1.0 \mu \mathrm{m}$, respectively. However, the grain diameters of the TNTZ-0.06O and TNTZ- -140 increase from 1.7 to $4.0 \mu \mathrm{m}$ and from 1.0 to $1.5 \mu \mathrm{m}$, respectively, with an increase in the heat-treatment time from 3.6 to $10.8 \mathrm{ks}$ for a heat-treatment temperature of $923 \mathrm{~K}$. Moreover, the diameters increase from 6.4 to $8.5 \mu \mathrm{m}$ and from 2.6 to $8.3 \mu \mathrm{m}$, respectively, with an increase in the heat-treatment time from 3.6 to $10.8 \mathrm{ks}$ for a heat-treatment temperature of $973 \mathrm{~K}$. Even though the grain diameters of the heat-treated TNTZ-0.06O and TNTZ-0.14O increase with an increase in the heat-treatment time and heat-treatment (a) TNTZ-0.06O

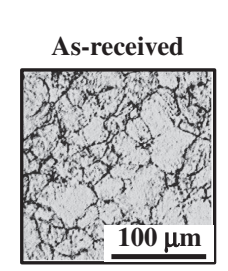

As-swaged
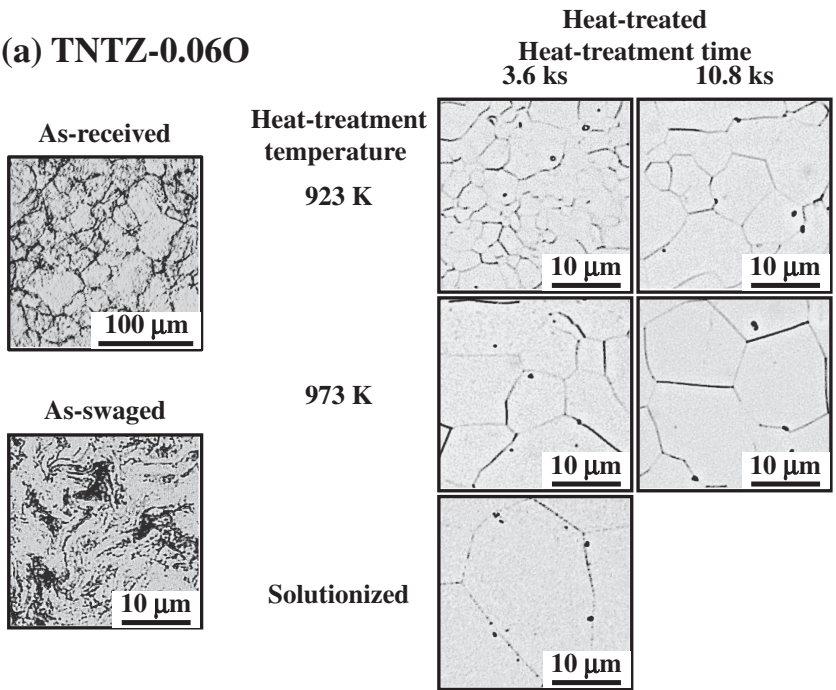

(b) TNTZ-0.140

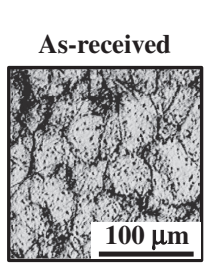

As-swaged

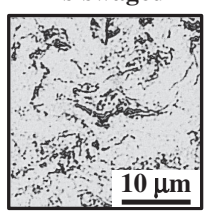

Fig. 2 Typical OM images of cross sections of as-received, as-cold swaged, heat-treated and solutionized (a) TNTZ-0.06O and (b) TNTZ0.140 bars.

(a) TNTZ-0.06O

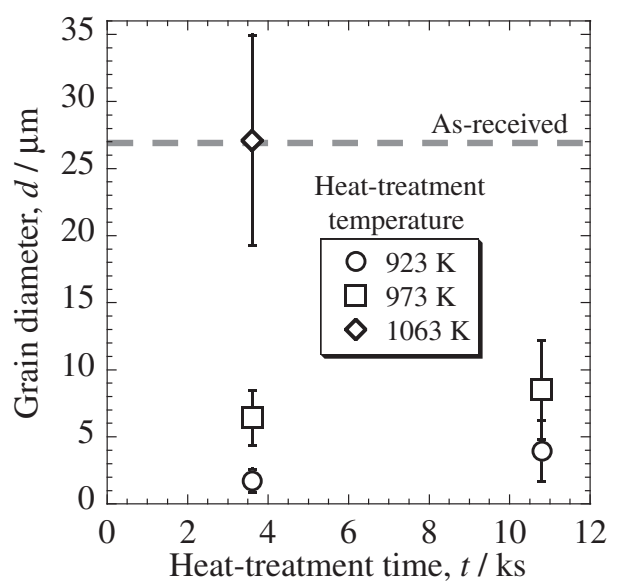

(b) TNTZ-0.140

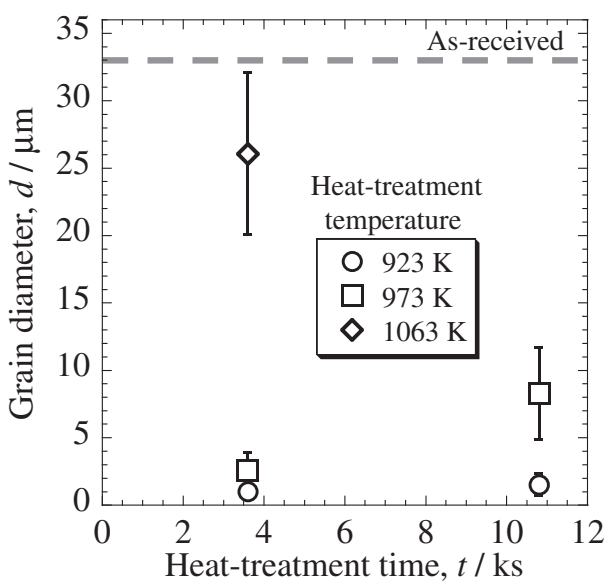

Fig. 3 Grain diameters of as-received, heat-treated and solutionized (a) TNTZ-0.06O and (b) TNTZ-0.14O quantified using OM images, as functions of the heat-treatment time. 
(a) TNTZ-0.060

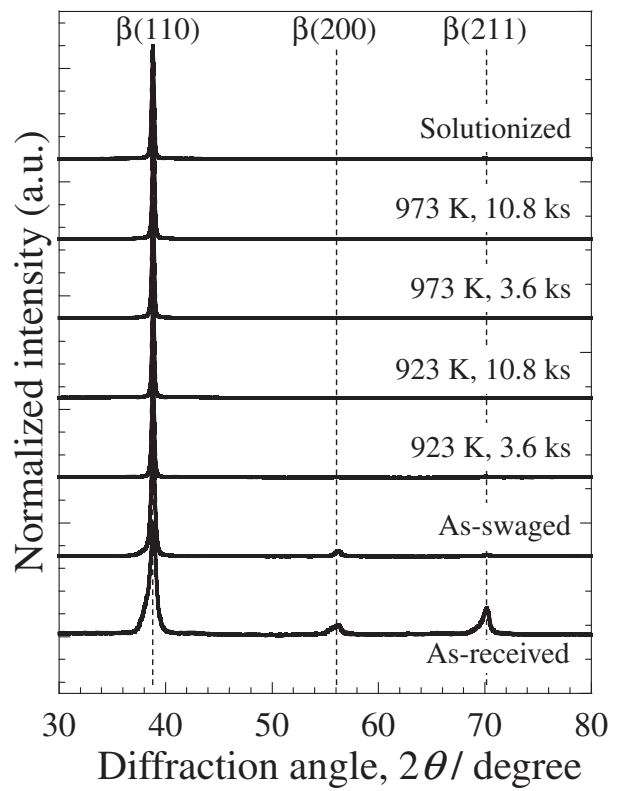

(b) TNTZ-0.140

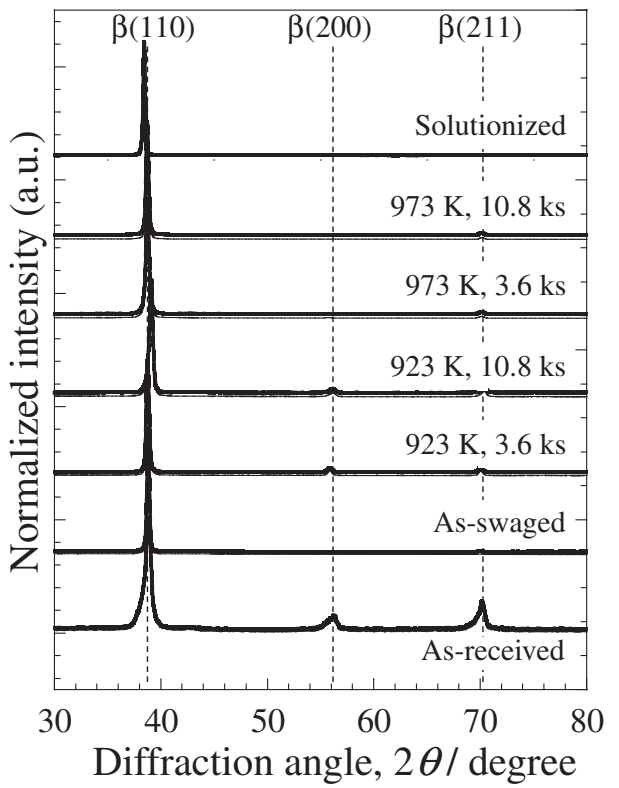

Fig. 4 Typical XRD profiles of cross sections of as-received, as-cold swaged, heat-treated and solutionized (a) TNTZ-0.06O and (b) TNTZ-0.140 bars.

(a) TNTZ-0.06O

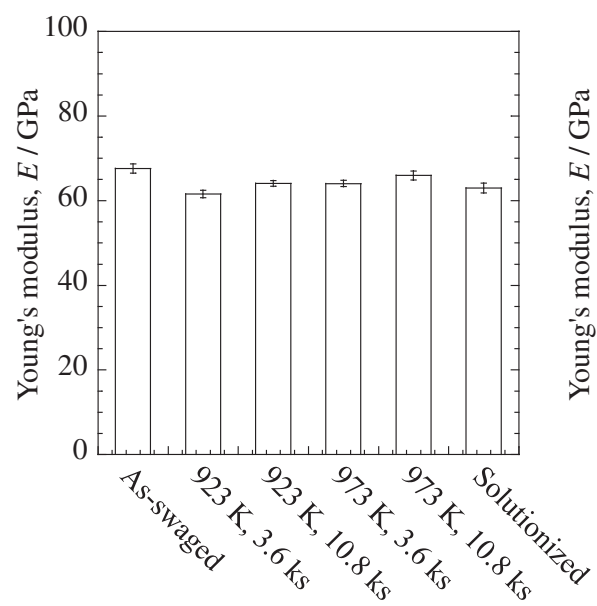

(b) TNTZ-0.140

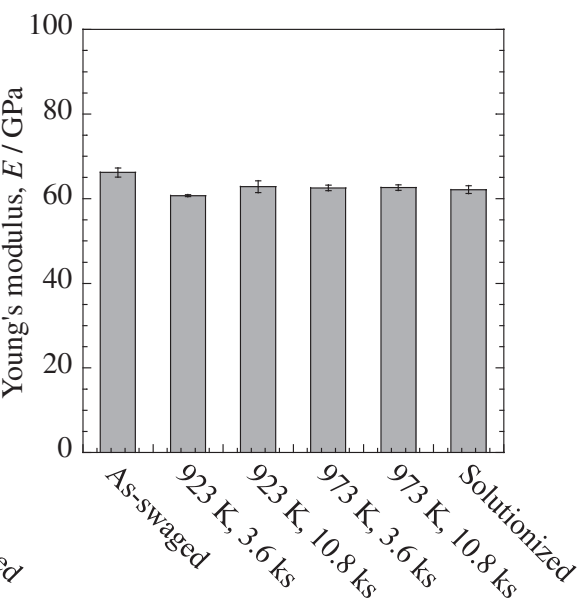

Fig. 5 Young's moduli of as-cold swaged, heat-treated and solutionized (a) TNTZ-0.06O and (b) TNTZ-0.14O.

temperature, they are still smaller than those of the asreceived and solutionized TNTZ-0.06O and TNTZ-0.14O. These results suggest that cold swaging, followed by heattreatment at 923 or $973 \mathrm{~K}$ for 3.6 or $10.8 \mathrm{ks}$, is effective method for achieving grain refinement in TNTZ.

Figure 4 shows typical XRD profiles of cross sections of the as-received, as-cold swaged, heat-treated and solutionized (a) TNTZ-0.06O and (b) TNTZ-0.14O bars. Here it is noted that these profiles were normalized using an intensity of each $\beta$ (110) peak. As shown in Fig. 4, only diffraction peaks attributable to $\beta$ (110), $\beta(200)$ and $\beta$ (211) are detected in the profiles of the TNTZ- $0.06 \mathrm{O}$ and TNTZ $-0.14 \mathrm{O}$. These results indicate that the as-received, as-cold swaged, heat-treated and solutionized TNTZ- $-0.06 \mathrm{O}$ and TNTZ- $-14 \mathrm{O}$ consist of single $\beta$ phase. Moreover, the intensities of the $\beta$ (200) and $\beta$ (211) peaks decrease significantly after the cold swaging process. This result suggests that the $\beta(110)$ texture develops as a result of the cold swaging process.

\subsection{Mechanical properties 3.2.1 Young's moduli}

Figure 5 shows the values of Young's moduli of the ascold swaged, heat-treated and solutionized (a) TNTZ-0.06O and (b) TNTZ-0.14O. As shown in Fig. 5, Young's moduli of the as-swaged TNTZ-0.06O and TNTZ-0.14O are 68 and $66 \mathrm{GPa}$, respectively. In addition, Young's moduli of the heattreated and solutionized TNTZ- $0.06 \mathrm{O}$ and TNTZ- $0.14 \mathrm{O}$ are 62-66 and 61-63 GPa, respectively. The differences of the Young's moduli between low and high oxygen contents and before and after the heat-treatment are very small. This is because, as above mentioned, these TNTZ alloys consist of single $\beta$ phase. Young's modulus of a metallic material mainly depends on its phase constitution. Therefore, the ascold swaged and heat-treated TNTZ-0.06O and TNTZ0.140 exhibited Young's moduli similar to, and as low as, that of the solutionized TNTZ-0.06O and TNTZ-0.14O, which have single $\beta$ phase. 
(a) TNTZ-0.06O

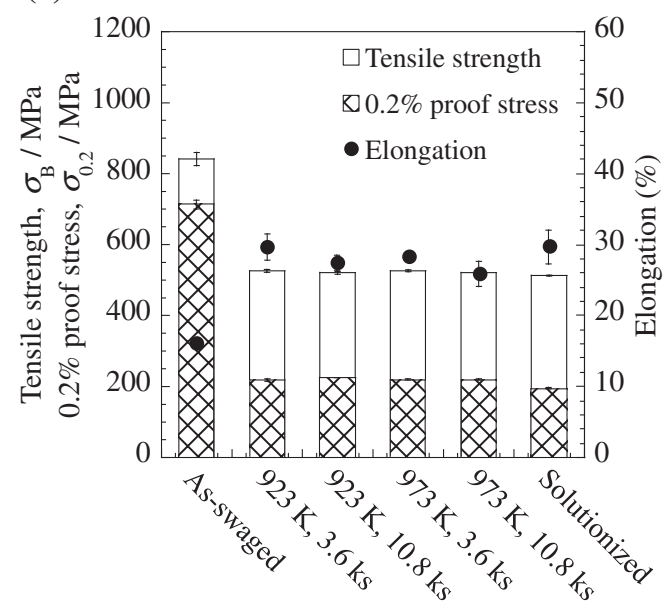

(b) TNTZ-0.140

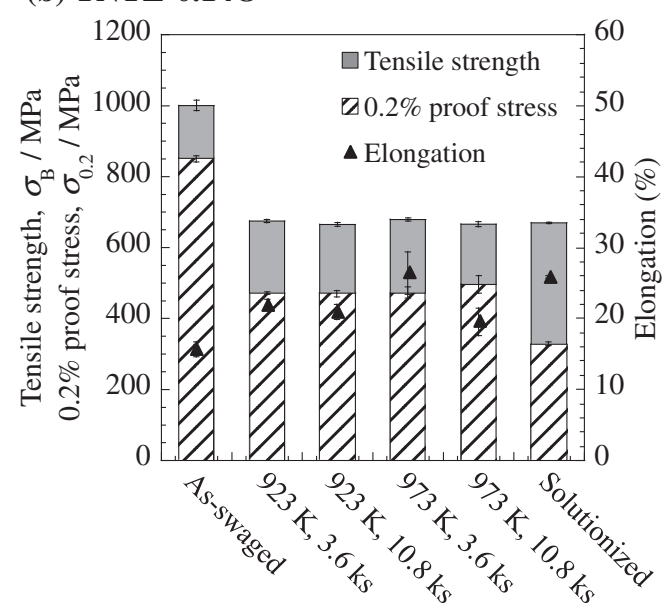

Fig. 6 Tensile strengths, $0.2 \%$ proof stresses and elongations of as-cold swaged, heat-treated and solutionized (a) TNTZ-0.06O and (b) TNTZ-0.14O.

\subsubsection{Tensile properties}

Figure 6 shows the values of the tensile strength $\left(\sigma_{\mathrm{B}}\right)$, $0.2 \%$ proof stress $\left(\sigma_{0.2}\right)$ and elongation of the as-cold swaged, heat-treated and solutionized (a) TNTZ-0.06O and (b) TNTZ-0.14O. As shown in Fig. 6, the tensile strengths of the as-swaged TNTZ-0.06O and TNTZ-0.14O are 841 and $1001 \mathrm{MPa}$, respectively. After the heat and solution treatments, the tensile strengths of the TNTZ-0.06O and TNTZ$0.14 \mathrm{O}$ decrease from 841 to $513-526 \mathrm{MPa}$ and from 1001 to 665-679 MPa, respectively. Moreover, the elongations of the as-swaged TNTZ-0.06O and TNTZ-0.14O are both $16 \%$. After the heat and solution treatments, the elongations of the TNTZ- $0.06 \mathrm{O}$ and TNTZ-0.14O increase from $16 \%$ to 26 $30 \%$ and to $20-26 \%$, respectively. The decrease in the tensile strengths and increase in the elongations of the TNTZ-0.06O and TNTZ- 0.140 after the heat and solution treatments are caused by the disappearance of dislocations, which are accumulated in the alloys during the cold swaging process, as a result of the heat and solution treatments.

The tensile strengths of the as-swaged, heat-treated and solutionized TNTZ- $0.14 \mathrm{O}$ are approximately $30 \%$ higher than those of TNTZ-0.06O. Moreover, the $0.2 \%$ proof stresses of the as-swaged, heat-treated and solutionized TNTZ-0.14O are approximately 20, 110 and $70 \%$ higher, respectively, than those of TNTZ-0.06O. However, the elongations of the heat-treated and solutionized TNTZ0.140 are approximately $20 \%$ lower than those of TNTZ$0.06 \mathrm{O}$. These results indicate that solid solution strengthening using oxygen has a significant effect on the tensile properties of TNTZ.

On the other hand, the dependence of the tensile properties of the TNTZ-0.06O and TNTZ- $0.14 \mathrm{O}$ on their grain diameters is not significant. Figure 7 shows the tensile strengths of the heat-treated and solutionized TNTZ- $-0.06 \mathrm{O}$ and TNTZ- 0.140 as functions of the inverse square root of their grain diameters $\left(d^{-1 / 2}\right)$ (Hall-Petch plots). As can be seen from the Fig. 7, these Hall-Petch plots have positive slopes. However, the values of the Hall-Petch constant $(k)$ for the TNTZ-0.06O $\left(k_{\text {TNTZ- }-0.06 \mathrm{O}}=0.02\right)$ and TNTZ-0.14O $\left(k_{\mathrm{TNTZ}-0.14 \mathrm{O}}=0.005\right)$ are much smaller than those of pure $\mathrm{Ti}$ $\left(k_{\mathrm{CP}-\mathrm{Ti}}=0.2\right)$ and another $\beta$-type $\mathrm{Ti}$ alloy (Ti-15.2Mo,

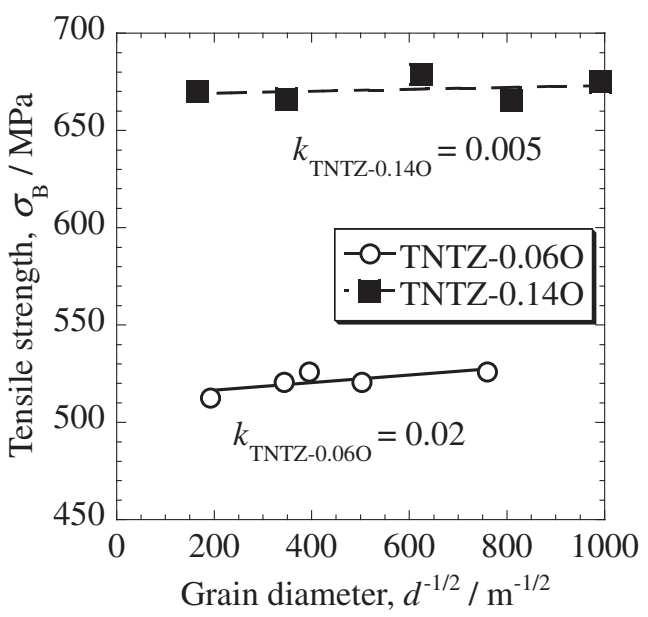

Fig. 7 Tensile strengths of heat-treated and solutionized (a) TNTZ-0.06O and (b) TNTZ-0.14O as functions of the inverse square root of their grain diameters $\left(d^{-1 / 2}\right)$ (Hall-Petch plots).

$\left.k_{\mathrm{Ti}-15.2 \mathrm{Mo}}=0.4\right) .{ }^{15)}$ These results indicate that the grain refinement caused by the cold swaging process and the subsequent heat treatment does not have a significant effect on the tensile properties of TNTZ. As above mentioned, the XRD profiles of the heat-treated TNTZ-0.06O and TNTZ$0.14 \mathrm{O}$ (Fig. 4) indicate that they have a strong $\beta(110)$ texture. Grain refinement strengthening is associated with a mismatch of grain orientations. Therefore, the orientation unit can be considered an effective grain. Even though grain refinement is caused by the cold swaging process and the subsequent heattreatment, the strong $\beta(110)$ texture suggests that the effective grain diameters of the TNTZ $-0.06 \mathrm{O}$ and TNTZ- $0.14 \mathrm{O}$ are significant larger than those determined using the OM images.

The $0.2 \%$ proof stresses for the heat-treated and solutionized TNTZ-0.06O are significantly lower than one-half of the corresponding tensile strengths. The ratios of the $0.2 \%$ proof stresses to the tensile strengths $\left(\sigma_{0.2} / \sigma_{\mathrm{B}}\right)$ are in the range of $38-43 \%$. On the other hand, except solutionized TNTZ0.140 , the $0.2 \%$ proof stresses of the heat-treated TNTZ0.140 are higher than half their corresponding tensile strengths. The ratios of the $0.2 \%$ proof stresses to the tensile strength $\left(\sigma_{0.2} / \sigma_{\mathrm{B}}\right)$ are in the range of $70-74 \%$. Duerig et al. 
(a) Heat-treated TNTZ-0.060 $923 \mathrm{~K}, 3.6 \mathrm{ks}$

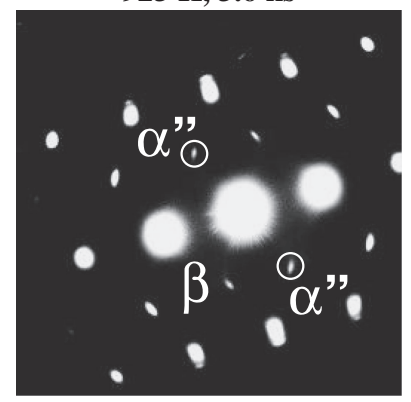

(b) Solutionized TNTZ-0.06O

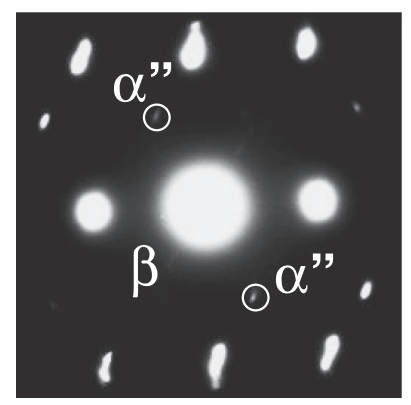

(c) Heat-treatedTNTZ-0.140 $923 \mathrm{~K}, 3.6 \mathrm{ks}$

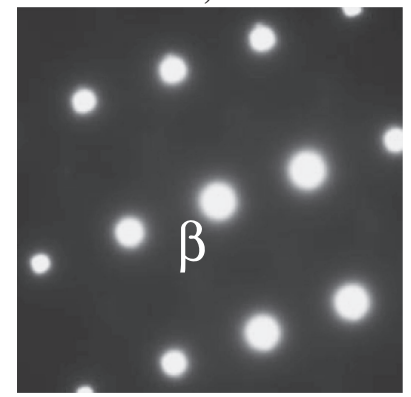

(d) Solutionized TNTZ-0.140

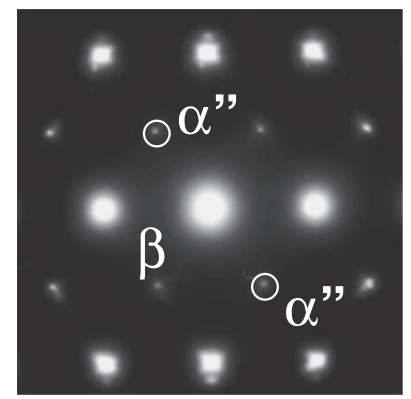

Fig. 8 Typical electron diffraction patterns of the $[110]_{\beta}$ zone of fractured sections of (a) heat-treated TNTZ-0.06O, (b) solutionized TNTZ-0.06O, (c) heat-treated TNTZ-0.14O and (d) solutionized TNTZ-0.14O after subjected to a tensile test.

have reported that the $0.2 \%$ proof stresses of $\mathrm{Ti}$ alloys decrease when a stress-induced $\alpha^{\prime \prime}$ martensite phase is formed. ${ }^{16)}$ Therefore, the microstructures of the fractured sections of the TNTZ-0.06O and TNTZ-0.14O were evaluated by TEM. Figure 8 shows typical electron diffraction patterns of the $[110]_{\beta}$ zone of the fractured sections of the (a) heat-treated TNTZ-0.06O, (b) solutionized TNTZ-0.06O, (c) heat-treated TNTZ-0.14O and (d) solutionized TNTZ$0.14 \mathrm{O}$ after subjected to the tensile test. The electron diffraction patterns of the fractured sections of the heattreated and solutionized TNTZ-0.06O as well as that of the solutionized TNTZ- $0.14 \mathrm{O}$ show spots attributable to the $\beta$ and $\alpha^{\prime \prime}$ martensite phases (Figs. 8(a), 8(b) and 8(d)). Moreover, a double yielding is observed in the stress-strain $(S-S)$ curves of the heat-treated and solutionized TNTZ-0.06O and the solutionized TNTZ-0.14O (not shown here). These results indicate that a stress-induced martensite phase transformation occurs in the heat-treated and solutionized TNTZ-0.06O and in the solutionized TNTZ-0.14O during tensile loading. On the other hand, the electron diffraction pattern of the fractured section of the heat-treated TNTZ-0.14O shows spots attributable only to the $\beta$ phase (Fig. 8(c)). Geng et al. have reported that oxygen can suppress the formation of the stressinduced $\alpha^{\prime \prime}$ martensite phase in TNTZ. ${ }^{14)}$ Moreover, Niinomi et al. have reported that the stress-induced $\alpha^{\prime \prime}$ martensite phase forms more readily in solutionized TNTZ than in heattreated ones. ${ }^{17)}$ Together, these results and reports suggest that the stress-induced martensite phase transformation does not occur in the heat-treated TNTZ- 0.140 during tensile loading. Therefore, the ratios of the $0.2 \%$ proof stresses to tensile strengths $\left(\sigma_{0.2} / \sigma_{\mathrm{B}}\right)$ of the heat-treated TNTZ- $0.14 \mathrm{O}$ are

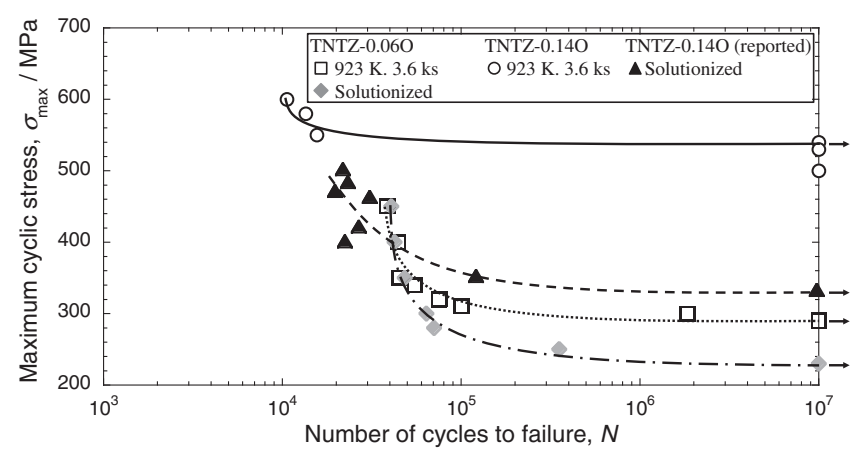

Fig. $9 S-N$ curves of TNTZ- $0.06 \mathrm{O}$ heat-treated at $923 \mathrm{~K}$ for $3.6 \mathrm{ks}$, the solutionized TNTZ-0.06O specimen and the TNTZ-0.14O specimen heat-treated at $923 \mathrm{~K}$ for $3.6 \mathrm{ks}$. The previously reported $S-N$ curve for a solutionized TNTZ alloy is also shown.

relatively higher than those of the heat-treated and solutionized TNTZ- $0.06 \mathrm{O}$ and the solutionized TNTZ-0.14O.

\subsubsection{Fatigue properties}

The $S-N$ curves of the TNTZ- $0.06 \mathrm{O}$ heat-treated at $923 \mathrm{~K}$ for $3.6 \mathrm{ks}$, the solutionized TNTZ-0.06O and the TNTZ$0.14 \mathrm{O}$ heat-treated at $923 \mathrm{~K}$ for $3.6 \mathrm{ks}$ are shown in Fig. 9; the previously reported $S-N$ curve for a solutionized TNTZ (Ti: bal, Nb: 31.5 , Ta: 11.6, $\mathrm{Zr}: 4.7$, O: 0.14 mass \%) is also shown. As can be seen from the Fig. 9, the difference in the fatigue strengths of the heat-treated and solutionized TNTZ$0.06 \mathrm{O}$ is very small in the low-cycle fatigue life region $\left(<1 \times 10^{5}\right.$ cycles $)$. This result is in agreement with the results of the tensile tests of the heat-treated and solutionized TNTZ0.06O. As shown in Fig. 6(a), the difference in the tensile properties of the heat-treated and solutionized TNTZ-0.06O is very small. On the other hand, the fatigue strength of the heat-treated TNTZ-0.06O is higher than that of the solutionized TNTZ-0.06O in high-cycle fatigue life region $\left(>1 \times 10^{5}\right.$ cycles $)$. Moreover, the fatigue strength of the heattreated TNTZ- 0.140 is much higher than that of previously reported solutionized TNTZ. The fatigue limits of the heattreated and solutionized TNTZ-0.06O are approximately 290 and $230 \mathrm{MPa}$, respectively. In addition, the fatigue limits of the heat-treated TNTZ- -140 is $540 \mathrm{MPa}$, while the value previously reported for solutionized TNTZ- 0.140 is $330 \mathrm{MPa}$. These results indicate that the gain refinement caused by cold swaging and a subsequent heat-treatment can improve the high-cycle fatigue life properties of TNTZ.

The typical XRD profiles of the fractured sections of the heat-treated and solutionized TNTZ- $0.06 \mathrm{O}$ as well as that of the heat-treated TNTZ-0.140 after subjected to a fatigue test are shown in Fig. 10. Diffraction peaks attributable to the $\alpha^{\prime \prime}$ (020) and $\alpha^{\prime \prime}(021)$ phases can clearly be observed in the profiles of the heat-treated and solutionized TNTZ-0.06O. It should be noted that the stress-induced $\alpha^{\prime \prime}$ martensite phase does not appear in the previously reported solutionized TNTZ after tensile loading. Li et al. have reported that the fatigue strength of $\beta$-Ti alloys decreases when stress gets concentrated at the interface between the stress-induced $\alpha^{\prime \prime}$ martensite phase and the $\beta$ phase. ${ }^{18)}$ Therefore, controlling the formation of the stress-induced $\alpha^{\prime \prime}$ martensite phase during cyclic loading is also important for improving the fatigue properties of TNTZ. As above mentioned, oxygen can suppress the formation of the stress-induced $\alpha^{\prime \prime}$ martensite 


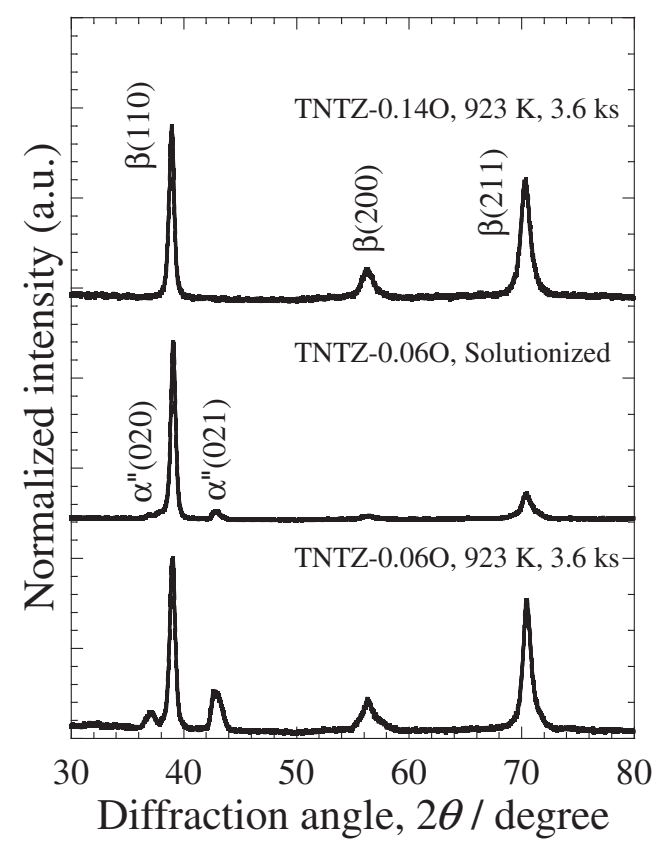

Fig. 10 Typical XRD profiles of fractured sections of heat-treated and solutionized TNTZ- $0.06 \mathrm{O}$ and heat-treated TNTZ-0.14O after subjected to a fatigue test.

phase in TNTZ. Moreover, solid solution strengthening owing to oxygen is expected to improve the fatigue properties of these alloys. Therefore, the fatigue properties of the heat-treated TNTZ-0.140 and the previously reported solutionized TNTZ are better than those of the heat-treated and solutionized TNTZ-0.06O. In particular, because the heat-treated TNTZ-0.14O is subjected to grain-refinement strengthening and solid-solution strengthening and because the formation of the stress-induced $\alpha^{\prime \prime}$ martensite phase is suppressed in it, it exhibits better fatigue properties than the heat-treated and solutionized TNTZ- $0.06 \mathrm{O}$ and the previously reported solutionized TNTZ-0.14O.

The results of this study indicate that grain refinement caused by cold swaging and a subsequent heat-treatment, and the addition of oxygen in the amount of 0.14 mass $\%$ can improve the tensile and fatigue properties of TNTZ without increasing their Young's moduli.

\section{Conclusions}

(1) After the TNTZ-0.06O and TNTZ-0.14O subjected to cold swaging and a subsequent heat-treatment at $923 \mathrm{~K}$ for $3.6 \mathrm{ks}$, their grain diameters decrease from $27 \mu \mathrm{m}$ (as-received) to $1.7 \mu \mathrm{m}$ and from $33 \mu \mathrm{m}$ (as-received) to $1.0 \mu \mathrm{m}$, respectively. In addition, the grain diameters of these TNTZ increase with an increase in the heattreatment time and temperature. However, the grain diameters of the heat-treated TNTZ-0.06O and TNTZ0.140 remained smaller than those of the as-received and solutionized TNTZ-0.06O and TNTZ-0.14O.

(2) The XRD profiles of cross sections of the as-received, as-cold swaged, heat-treated and solutionized TNTZ$0.06 \mathrm{O}$ and TNTZ- $0.14 \mathrm{O}$ bars indicate that these TNTZ- $0.06 \mathrm{O}$ and TNTZ- $0.14 \mathrm{O}$ consist of a single $\beta$ phase. Moreover, the $\beta$ (110) texture develops as a result of cold swaging.

(3) Young's moduli of the as-cold swaged, heat-treated and solutionized TNTZ-0.06O and TNTZ-0.14O are within the range of $61-68 \mathrm{GPa}$. The differences of the Young's moduli between low and high oxygen contents and before and after the heat-treatment are very small.

(4) The tensile strengths and elongations of the as-cold swaged, heat-treated and solutionized TNTZ-0.140 are approximately $30 \%$ higher and $20 \%$ lower, respectively, than those of the corresponding TNTZ-0.06O.

(5) The values of the Hall-Petch constant $(k)$ for TNTZ-0.06O $\left(k_{\text {TNTZ- } 0.060}=0.02\right)$ and TNTZ-0.14O $\left(k_{\mathrm{TNTZ}-0.14 \mathrm{O}}=0.005\right)$ are much smaller than those for pure $\mathrm{Ti}\left(k_{\mathrm{CP}-\mathrm{Ti}}=0.2\right)$ and another $\beta$-type Ti alloy $(\mathrm{Ti}-$ $\left.15.2 \mathrm{Mo}, k_{\mathrm{Ti}-15.2 \mathrm{Mo}}=0.4\right)$.

(6) The heat-treated TNTZ-0.14O has significantly better fatigue properties (its fatigue limit is $540 \mathrm{MPa}$ ) than heat-treated, solutionized TNTZ-0.06O and a previously reported solutionized TNTZ-0.14O.

\section{Acknowledgements}

This study was supported in part by a Grant-in-Aid for Scientific Research (A) 24246111 from the Japan Society for the Promotion of Science (JSPS) and by the Inter-University Cooperative Research Program "Innovation Research for Biosis-Abiosis Intelligent Interface" of the Ministry of Education, Culture, Sports, Science and Technology, Japan.

\section{REFERENCES}

1) R. Huiskes, H. Weinans and B. van Rietberge: Clin. Orthop. Relat. Res. 274 (1992) 124-134.

2) D. Kuroda, M. Niinomi, M. Morinaga, Y. Kato and T. Yashiro: Mater. Sci. Eng. A 243 (1998) 244-249.

3) Y. L. Hao, S. J. Li, S. Y. Sun, C. Y. Zheng, Q. M. Hu and R. Yang: Appl. Phys. Lett. 87 (2005) 091906.

4) T. Akahori, M. Niinomi, H. Fukui, M. Ogawa and H. Toda: Mater. Sci. Eng. C 25 (2005) 248-254.

5) P. K. Zysset, X. E. Guo, C. E. Hoffler, K. E. Moore and S. A. Goldstein: J. Biomech. 32 (1999) 1005-1012.

6) E. Bertrand, T. Gloriant, D. M. Gordin, E. Vasilescu, P. Drob, C. Vasilescu and S. I. Drob: J. Mech. Behav. Biomed. Mater. 3 (2010) 559-564.

7) R. R. Boyer, G. E. Welsch and E. W. Collings (Eds.): Materials Properties Handbook: Titanium Alloys, (ASM International, Materials Park, OH, 1994) pp. 483-636.

8) T. Akahori, M. Niinomi, K. Fukunaga and I. Inagaki: Metall. Mater. Trans. A 31 (2000) 1937-1948.

9) M. Nakai, M. Niinomi and T. Oneda: Metall. Mater. Trans. A 43 (2012) 294-302.

10) C. Ouchi, H. Iizumi and S. Mitao: Mater. Sci. Eng. A 243 (1998) 186195.

11) S. Nagarjuna, M. Srinivas, K. Balasubramanian and D. S. Sarma: Acta Mater. 44 (1996) 2285-2293.

12) E. Pink and J. R. Arsenault: Metall. Sci. 6 (1972) 1-6.

13) H. W. Rosenberg and W. D. Nix: Metall. Trans. 4 (1973) 1333-1338.

14) F. Geng, M. Niinomi and M. Nakai: Mater. Sci. Eng. A 528 (2011) 5435-5445.

15) K.-H. Chia, K. Jung and H. Conrad: Mater. Sci. Eng. A 409 (2005) 3238.

16) T. W. Duerig, G. T. Terlinde and J. C. Williams: Metall. Trans. A 11 (1980) 1987-1998.

17) M. Niinomi, T. Akahori, M. Nakai and H. Tsutsumi: Mater. Trans. 50 (2009) 1704-1712.

18) S. J. Li, T. C. Cui, Y. L. Hao and R. Yang: Acta Biomater. 4 (2008) 305-317. 